\title{
INTERACTION OF 3-HYDROXY PYRIDINE AND SURFACTANT MICELLES: A FLUORESCENCE STUDIES
}

\author{
ANSHU MAHLAWAT*, ARUN GOYAL \\ Department of Chemistry, Government P.G. College Rajgarh, Alwar, Rajasthan, India. Email: anshumahlawat19@gmail.com
}

Received: 25 April 2021, Revised and Accepted: 07 June 2021

\begin{abstract}
Objective: Micellar solubilization is a powerful alternative for dissolving hydrophobic compound in aqueous environment. 3-hydroxy pyridine (3HP) derivatives are the potential endogenous photosensitizers. 3-HP derivatives show protective effect in clinical extreme condition such as hypoxia, hyperthermia, hypokinesia. Micellization of 3-HP followed by solubilization would catalyze its pharmaceutical activities which may serve better results in medicinal and analytical fields.
\end{abstract}

Methods: Fluorescence and absorption spectroscopy techniques are used to monitor the micellar solubilization studies of 3-HP. Solubilization studies of 3 -HP with various anionic, cationic and nonionic surfactants have been performed in aqueous medium around $23-25^{\circ} \mathrm{C}$ temperature. The solubilization action of the surfactant has also been determined by theoretical calculated spectral parameters such as empirical fluorescence coefficient, quantum yield, stokes, shift and molar absorption coefficient.

Results: 3-HP shows fluorescence excitation peak at $315 \mathrm{~nm}$ and emission peak at $390 \mathrm{~nm}$ respectively while the absorbance of 3-HP has been found to be maximum at $305 \mathrm{~nm}$. The fluorescence as well as the theoretically calculated spectral data has been used to characterize the hetero environment of the micelles in terms of their polarity, probe solubilization site and critical micelle concentration.

Conclusion: This article briefly discusses the importance of surfactants in biological system model as well as the use of micelles in pharmacy as an important tool that finds numerous applications.

Keywords: 3-hydroxy pyridine, Fluorescence, Micelles, Solubilization.

(C) 2021 The Authors. Published by Innovare Academic Sciences Pvt Ltd. This is an open access article under the CC BY license (http://creativecommons.org/ licenses/by/4.0/) DOI: http://dx.doi.org/10.22159/ajpcr.2021v14i8.41895. Journal homepage: https://innovareacademics.in/journals/index.php/ajpcr

\section{INTRODUCTION}

Fluorescence spectroscopy is a well-established extensively used research and analytical tool in many disciplines [1]. Fluorescence spectroscopy can also serve as fantastic tool to study the micellization of surfactants due to its excellent sensitivity towards the environment surrounding the fluorophore which exhibits different fluorescence characteristics depending on the properties of the solubilizing medium. In recent years, a remarkable growth in the use of fluorescence in food analysis, biotechnology, drug delivery and design and clinical diagnostics of disease have been observed and several reports are given on its applications [2-6].

Micellization is an important phenomenon not only because a number of important interfacial phenomena, such as detergency and solubilization, depend on the existence of micelles in solution but also because it affects other interfacial phenomena, such as surface or interfacial tension reduction, that do not directly involve micelles. Micelles have been the subject to the numerous investigations due to their importance as model system for mimicking bio membranes [7-9]. Many characteristics of molecules, for example, absorption and fluorescence spectra, deprotonation and protonation equilibrium etc. are changed drastically in micellar media [10-13]. Conversely, changes observed in the absorption spectra of molecules have been utilized to study the properties of micelles, such as critical micelle concentration (CMC), viscosity, polarity of different sites [14]. Micelles also involve in drug delivery, to minimize drug degradation and loss, to prevent harmful side effects, and to increase drug bioavailability.

${ }^{13} \mathrm{C}$ and ${ }^{1} \mathrm{H}$ NMR spectra of 3-hydroxy pyridine (3-HP) and its derivatives were analyzed [15]. The protective effect of 3-HP derivatives in various extreme conditions such as hypoxia, hypothermia, and hypokinesia have been investigated [16]. Koval'chukova et al. [17] studied the physicochemical properties of some complex compounds of 3-HP and transition metals. Bromido and Chlorido complexes of $\mathrm{Cu}$ with 3-HP were prepared and the magnetic properties of complexes were analyzed by the infrared, ultraviolet/visible and electron paramagnetic resonance spectra [18]. Bridges et al. [19] were investigated that 2 and 4-HP were non-fluorescent at all $\mathrm{p}^{\mathrm{H}}$ values while 3-HP were fluorescent and studied the variations of the excitation and fluorescence wavelength and fluorescence intensity at different $\mathrm{p}^{\mathrm{H}}$ values. The anticancer properties of 3-HP and platinum complexes were studied and their activity against ovarian cancer cell lines have been determined [20].

\section{METHODS}

Analytically pure 3-HP used was a Loba sample. The following surfactants were employed; (a) Nonionic: (i) Tx-100: Polyoxyethylene tert-octyl phenyl ether, (ii) Tween-80: Polyoxyethylene sorbitain monooleate, (iii) Tween-20: Polyoxyethylene sorbitain monolaurate. (b) Anionic: (i) SLS: Sodium lauryl sulphate, (ii) DBSS: Dodecylbenzyl sodium sulphonate, (iii) DSSS: Dioctyl sodium sulphosuccinate. (c) Cationic: (i) CPC: Cetylpyridinum chloride, (ii) CTAB: Cetyltrimethyl ammonium bromide, (iii) MTAB: Myristyltrimethyl ammonium bromide. All the surfactants used were either of Sigma (USA) or BDH (UK) products. The stock solution of 3-HP was prepared in double distilled water. All the experiments were performed around $23-25^{\circ} \mathrm{C}$ in aqueous medium keeping the final concentration of $3-\mathrm{HP}$ at $6^{*} 10^{-5} \mathrm{M}$ for fluorescence studies. For absorption studies the concentration of 3-HP was kept at $2 * 10^{-4} \mathrm{M}$ throughout the experiments.

All the fluorimetric experiments were carried out with Perkin Elmer Fluorescence Spectrophotometer (Model no. 204A) with a synchronized strip chart recorder (Model no. 056). A Xenon lamp was used as a light 
source. For recording the fluorescence excitation and emission spectra, its slit width was kept at $10 \mathrm{~nm}$ and a cell of $1 \mathrm{~cm}$ path length was used. The absorption measurements were made with Hewlett Packard 8452, and diode array spectrophotometer.

The purity of the surfactants was checked by determining their CMC values the help of surface tension measurements, employing drop-weight method. The values obtained coincided with the recorded values. The absolute fluorescence quantum yield $\left(\Phi_{\mathrm{f}}\right)$ of 3-HP was calculated relative to anthracene solution as standard. Fluorescence emission of anthracene is in the same range as that of 3-HP. Approximate corrections were made to compensate for different absorption of the compound and the standard. Each time the total intensity of fluorescence emission was measured for the standard and the sample from the area of the fluorescence spectrum recorded over the whole range of emission under identical conditions. Molar extinction coefficient data have been reported as its logarithm $(\log \varepsilon)$. The Stokes' shift data have also been calculated and are expressed in nanometers.

\section{RESULTS}

\section{Fluorescence studies}

The aqueous solution of 3-HP has been showed maximum excitation peak at $315 \mathrm{~nm}$ and maximum emission peak at $390 \mathrm{~nm}$. On addition of Tx-100 the fluorescence intensity decreased significantly as a consequence of fluorescence quenching without any appreciable change in the shape of the emission band. Tween-20 and Tween-80 increased the fluorescence intensity. Tween-20 caused maximum enhancement in fluorescence intensity with a red shift of $10 \mathrm{~nm}$. The fluorescence spectral changes on addition of Tween-20 are as given in Fig. 1. The fluorescence intensity of 3-HP increased on adding anionic surfactants to it. All the anionic surfactants caused a blue shift of 10$30 \mathrm{~nm}$ in peak position. The cationic surfactants CTAB and MTAB caused an enhancement in fluorescence intensity with a blue shift of $5 \mathrm{~nm}$ and $30 \mathrm{~nm}$ respectively, while CPC decreased the fluorescence intensity. The fluorescence intensity in absence and presence of nonionic, anionic and cationic surfactants is given in Table 1.

\section{Absorption and quantitative studies}

The absorbance of 3-HP was found to be maximum at $305 \mathrm{~nm}$. Tween-20 and Tween-80 increased the absorbance with a red shift of 5-10 nm. TX-100 decreased the absorbance with a red shift of $5 \mathrm{~nm}$. Anionic and cationic surfactants caused an enhancement in the absorbance with a blue shift of 5-15 nm. The fluorescence quantum yield $\left(\Phi_{\mathrm{f}}\right)$ values of surfactant added 3-HP have been calculated. Data obtained from calculation show the increasing order in quantum yield values for all the nonionic, cationic and anionic surfactants except TX-100 and CPC. Molar extinction coefficient $(\log \varepsilon)$ values for all surfactants showed an increasing trend except Tx-100. Empirical fluorescence coefficient $\left(\mathrm{K}_{\mathrm{f}}\right)$ values of 3-HP with different surfactants were found to be parallel with the fluorescence intensity of the compound. All the theoretically calculated spectral parameters are listed in Table 2.

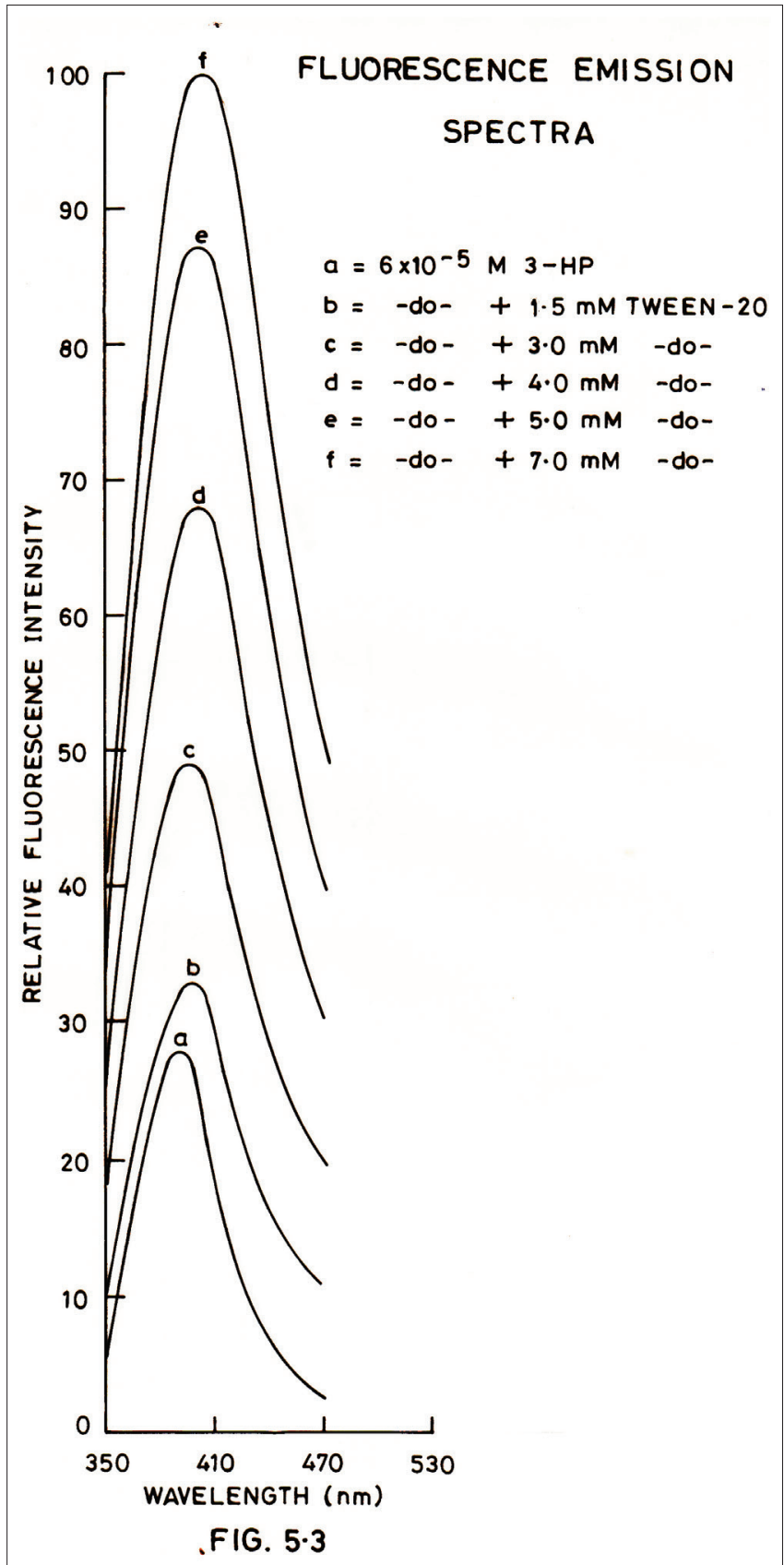

Fig. 1: Influence of addition of Tween-20 on fluorescence intensity of $6 \times 10^{-5} \mathrm{M}$, 3-hydroxy pyridine solution. (a) No Surfactant;

(b) $1.5 \mathrm{mM}$ Tween-20; (c) $3.0 \mathrm{mM}$ Tween-20; (d) $4.0 \mathrm{mM}$,

Tween-20; (e) 5.0 mM Tween-20; (f) 7.0 mM Tween-20

Table 1: Fluorescence intensity of $3-\mathrm{HP}$ in absence and presence of surfactant $\lambda_{\mathrm{ex}}=315 \mathrm{~nm}, \lambda_{\mathrm{em}}=390 \mathrm{~nm}, \mathrm{P} . \mathrm{M}$. Gain=3 Sensitivity Range=3

\begin{tabular}{|c|c|c|c|c|c|}
\hline $\begin{array}{l}\text { Name of } \\
\text { surfactant }\end{array}$ & $\begin{array}{l}\text { Relative fluorescence intensity } \\
\text { in absence of surfactant }\end{array}$ & $\begin{array}{l}\text { CMC'S of } \\
\text { surfactant (mM) }\end{array}$ & $\begin{array}{l}\text { Maximum Concentration } \\
\text { of Surfactant used (mM) }\end{array}$ & $\begin{array}{l}\text { Relative fluorescence intensity } \\
\text { in presence of surfactant }\end{array}$ & $\lambda_{\mathrm{em}}(\mathrm{nm})$ \\
\hline Tx-100 & 28 & 0.26 & 1.5 & 10 & 390 \\
\hline Tween-80 & 28 & 0.1 & 6.0 & 94 & 390 \\
\hline Tween-20 & 28 & 0.05 & 7.0 & 100 & 400 \\
\hline $\mathrm{CPC}$ & 28 & 0.6 & 9.0 & 22 & 390 \\
\hline CTAB & 28 & 0.90 & 9.0 & 33 & 385 \\
\hline MTAB & 28 & 3.6 & 7.0 & 85 & 360 \\
\hline DSSS & 28 & 0.91 & 9.0 & 42 & 365 \\
\hline DBSS & 28 & 0.81 & 0.8 & 65 & 360 \\
\hline
\end{tabular}

3-HP: 3-Hydroxy pyridine, CMC: Critical micelle concentration, CPC: Cetylpyridinum chloride, CTAB: Cetyltrimethyl ammonium bromide, MTAB: Myristyltrimethyl ammonium bromide, SLS: Sodium lauryl sulphate, DSSS: Dioctyl sodium sulphosuccinate, DBSS: Dodecylbenzyl sodium sulphonate 
Table 2: Absorption maxima $\lambda_{\alpha}$, fluorescence maxima $\lambda_{\text {em }}$, molar extinction coefficient $(\log \varepsilon)$ and quantum yield $\left(\Phi_{f}\right)$ of 3-HP at different concentration of Tween-20

\begin{tabular}{|c|c|c|c|c|}
\hline $\begin{array}{l}\text { Concentratin } \\
\text { of Tween-20 } \\
\text { (Mm) }\end{array}$ & $\begin{array}{l}\text { Absorption } \\
\text { maxima } \lambda_{\alpha}\end{array}$ & $\begin{array}{l}\text { Molar extinction } \\
\text { coefficient }(\log \varepsilon \\
\left(\mathrm{dm}^{3} \mathrm{~mol}^{-1} \mathrm{~cm}^{-1}\right)\end{array}$ & $\begin{array}{l}\text { Fluorescene } \\
\text { Maxima } \lambda_{\mathrm{em}} \\
(\mathrm{nm})\end{array}$ & $\begin{array}{l}\text { Quantum } \\
\text { yield } \Phi_{\mathrm{f}}\end{array}$ \\
\hline 0.00 & 305 & 3.3607 & 390 & 0.2418 \\
\hline 1.5 & 305 & 3.4608 & 395 & 0.2968 \\
\hline 3.0 & 305 & 3.5409 & 395 & 0.3920 \\
\hline 4.0 & 310 & 3.5693 & 400 & 0.5084 \\
\hline 5.0 & 310 & 3.6159 & 400 & 0.5914 \\
\hline 7.0 & 315 & 3.6776 & 400 & 0.6055 \\
\hline
\end{tabular}

3-HP: 3-hydroxy pyridine

\section{Stokes' shift}

The Stokes' shift values of 3-HP were determined for different concentrations at room temperature. The Stokes' shift values increased on diluting the 3-HP solution. The calculated values are given in Table 3.

\section{DISCUSSION}

Tx-100 quenched fluorescence intensity because effective hydrogen bonding does not take place between solubilizate and surfactant micelles. The quenching also indicates that these compounds prefer the hydrophobic core to the hydrophilic poly(ethylene oxide) shell, particularly for Tx-100. Evidently the fluorescence of compounds is significantly weakened in the core such as in non-aqueous solvents. This implies that compound is embedded in the core is not hydrated around the aromatic rings [21]. Quenching can also be caused by nonradiation loss of energy from the excited molecules. Fluorescence quenching was also observed by the addition of CPC, which may be attributed to the electrostatic preferential interaction between the polar substituent of 3-HP molecules and the cationic head group of the surfactant which may result in change in the geometry of the solubilizate molecule, where it loses the coplanarity. The quenching may also be due to interaction between the ח-electron system of the excited state fluorophore and quencher molecule CPC due to the presence of nucleophilic pyridine ring in the structure which makes it act as a quencher through hydrogen bond between the proton donor and accepter. This will result in delocalization of the $\pi$-electrons of the excited state and hence loss of fluorescence [22].

On addition of anionic surfactants, a continuous enhancement in the fluorescence intensity was observed for 3-HP. The increase in fluorescence intensity and quantum yield suggests that they have solubilized suspended molecules, which are dispersed as macrocrystals in water which collide with anionic micelles to penetrate into the micellar core interior. Here the anionic micelles have formed 1:1 complex with the protonated solubilized molecules. This complex is called ion association complex [23].

Absorption spectra of 3-HP are very less affected in micellar media as compared to the fluorescence spectra. This may be because absorption is less sensitive to its environment as compared to fluorescence. No major change in the nature of absorption spectrum indicates no structural changes due to complex formation or dissociation or hydrogen bonding between 3-HP in the ground state and the surfactant. Blue shift obtained in maxima may be due to the difference in salvation energy of the excited state. The sufficiently large value of $\log \varepsilon$ are assigned to the $\pi \rightarrow \pi^{*}$ transitions.

The increase in fluorescence intensity and quantum yield values in ionic micellar media clearly indicate that the rates of non-radiative processes are less in micellar system in comparison to those in water. This could be due to the decrease in intersystem crossing rate [24]. Another reason for the increase in values could be due to absorption of fluorophore at the micellar surface, which decrease the rate of collisional deactivation
Table 3: Stokes' shift data of 3-HP at room temperature

\begin{tabular}{llllll}
\hline $\begin{array}{l}\text { Concentration } \\
\text { of } \mathbf{3 - H P}(\mathbf{M})\end{array}$ & F.I & $\boldsymbol{\lambda}_{\text {ex }}(\mathbf{n m})$ & F.I & $\boldsymbol{\lambda}_{\text {em }}(\mathbf{n m})$ & $\begin{array}{l}\text { Stokes' shift } \\
\left.\mathbf{( c m}^{-1}\right)\end{array}$ \\
\hline $1 \times 10^{-3}$ & 22 & 330 & 37 & 390 & 4662 \\
$7 \times 10^{-4}$ & 20 & 330 & 36 & 390 & 4662 \\
$5 \times 10^{-4}$ & 19 & 325 & 33 & 390 & 5128 \\
$3 \times 10^{-4}$ & 51 & 320 & 89 & 390 & 5608 \\
$1 \times 10^{-4}$ & 28 & 315 & 47 & 390 & 6105 \\
$7 \times 10^{-5}$ & 20 & 315 & 30 & 390 & 6105 \\
$6 \times 10^{-5}$ & 17 & 315 & 28 & 390 & 6105 \\
$5 \times 10^{-5}$ & 15 & 315 & 23 & 390 & 6105 \\
$3 \times 10^{-5}$ & 12 & 315 & 17 & 390 & 6105 \\
\hline $3-3-h y$
\end{tabular}

3-HP: 3-hydroxy pyridine

of fluorophore by water molecule. Greater quantum yields imply greater efficiency of fluorescence pathway.

Large Magnitude of Stokes' shift data obtained in the solubilizate molecules of 3-HP suggests the preferential solvation of the solute in the protic solvent to increase hydrogen bonding interaction. Structural transitions can be induced in charged micelles by increasing ionic strength of the medium. These large Stokes' shift values are due to hydrogen bond formation between the solute and the solvent in ground state. This bond breaks following excitation to $\mathrm{S}_{1}$ but reforms following proton transfer [25].

\section{CONCLUSION}

The present analysis indicates that during solubilization of solubilizate 3-HP into the surfactant system, the incorporation of the solubilizate influence the balance of favorable and unfavorable forces guiding micellization and structural changes occurring due to aggregation, dissociation and hydrogen bonding. Hence the process of micellization followed by solubilization of 3-HP would catalyze its pharmaceutical activities which may serve better results in medicinal and analytical fields. Thus one can generalize the physical understanding to study the phenomenon of micellar solubilization

\section{ACKNOWLEDGMENT}

The authors are thankful to the Head, Department of Chemistry, Jai Narain Vyas University, Jodhpur for providing necessary research facilities.

\section{AUTHOR'S CONTRIBUTION}

Anshu Mahlawat has performed the Solubilization, Fluorescence and absorption spectral study of the compound. Arun Goyal has been contributed in spectral study and data analysis. Both the authors draft the final manuscript.

\section{CONFLICTS OF INTEREST}

The authors declare that they have no conflicts of interests.

\section{AUTHOR'S FUNDING}

The author is also thankful to the CSIR, New Delhi for providing financial support.

\section{REFERENCES}

1. Naresh K. Applications of fluorescence spectroscopy. J Chem Pharm Sci 2014;5:18-21.

2. Diamandis EP. Immunoassays with time-resolved fluorescence spectroscopy: Principles and applications. Clin Biochem 1988;21:139-50.

3. Lakowicz JR. Topics in Fluorescence Spectroscopy: Biochemical Application. Vol. 3. New York: Plenum Press; 1992.

4. Davis LM, Williams PE, Ball DA, Swift KM, Matsyoshi ED. Data reduction methods for application of fluorescence correlation spectroscopy to pharmaceutical drug discovery. Curr Pharm Biotech 
2003;4:451-62.

5. Aslan K, Holley P, Geddes CD. Microwave accelerated metalenhanced fluorescence (MAMEF) with silver colloids in 96-well plates: Application to ultra-fast and sensitive immunoassays, high throughput screening and drug discovery. J Immunol Methods 2006;312:137-47.

6. Oswald B, Gruber M, Bohmer M, Lehmann F, Wolfbeis OS. Novel diode laser-compatible fluorophores and their application to single molecule detection, protein labeling and fluorescence resonance energy transfer immunoassay. Photochem Photobiol 2001;74:237-45.

7. Fendler JH. Membrane Mimetic Chemistry. New York: Wiley Intersciences Publications; 1982.

8. Scaiano JC, Abuin EB, Stewart LC. Photochemistry of benzophenon in micelles: Formation and decay of radical pairs. J Am Chem Soc 1982;104:5673-9.

9. Mittal KL, Lindman B. Surfactant in Solutions. New York: Plenum Press; 1984.

10. Turro NJ, Chow MF, Chung CJ, Tung CH. An efficient, high conversion photoinduced emulsion polymerization. Magnetic field effects on polymerization efficiency and polymer molecular weight. J Am Chem Soc 1980;102:7391-3.

11. Almegren K, Grieser F, Thomas JK. energy transfer from triplet aromatic hydrocarbons to $\mathrm{Tb}^{3+}$ and $\mathrm{Eu}^{3+}$ in aqueous micellar solutions. J Am Chem Soc 1979;101:2021-6.

12. Jay J, Johnston LJ, Scaiano JC. Quenching of pyrene fluorescence by cupric ions in micellar solution effect of quenching on the polarity reported by the probe. Chem Phys Lett 1988;148:517-22.

13. Moriya T. Excited-state reactions of coumarins, vii. the solventdependent fluorescence of 7-hydroxy coumarins. Bull Chem Soc Jpn 1988;61:1873-86

14. Kano K, Ueno Y, Hashimoto Y. Fluorescence studies on the characterization and solubilizing abilities of sodium dodecyl sulfate, hexadecyltrimethyammonium chloride and TritonX-100 micelles. J Phys Chem 1985;89:3161-6.

15. Vogeli U, Philipsborn WV. ${ }^{13} \mathrm{C}$ and ${ }^{1} \mathrm{H}$ NMR spectroscopic studies on the structure of N-methyl -3-pyridone and 3-hydroxy pyridine. Org
Magn Reson 1973;5:551-9.

16. Iasnetov VV. Protective effect of 3-hydroxypyridine derivatives in extreme conditions. Avia Ekal Med 2007;41:5-12

17. Koval'chukova OV, Strashnova SB, Zaitsev BE, Vovk TV. Synthesis and physicochemical properties of some transition metal complexes with 3-hydroxy pyridine. Russ J Coord Chem 2002;28:767-70.

18. Segedin P, Dolnicar U, Cuskic M, Jaglicic Z, Golobic A, Kozlevcar B. Halogenido analysis of structurally diverse complexes with 3-hydroxypyridine. Acta Chim Slov 2008;55:992-8.

19. Bridges JW, Davies DS, Williams RT. Fluorescence studies of some hydroxypyridines including compounds of the Vitamin b6 group. J Biochem 1966;98:451-68.

20. Abdullah A, Huq F, Chowdhary A, Tayyem H, Beale P, Fischer K. Studies on the synthesis, characterization, binding with DNA and activities of two cis-planaramineplatinum(II) complexes of the form: cis-PtL( $\left.\mathrm{NH}_{3}\right)$ $\mathrm{CL}_{2}$ where $\mathrm{L}=3$-hydroxypyridine and 2,3-diaminopyridine. BMC Chem Biol 2006;6:1-9.

21. Kanzaki R, Umebayashi Y, Maki T, Ishiguro S. Thermodynamics and fluorescence spectra of 1,10-phenanthroline in micelles of poly(ethylene oxide)-type nonionic surfactant. J Solution Chem 2004;33:699-709.

22. Shizuka H, Fukushima M, Fuju T, Kobayashi T, Ohtani H, Hoshino M. Proton-induced quenching of methoxynaphthalenes studied by laser flash photolysis and inclusion effect of $\beta$-cyclodextrin on the quenching. Bull Chem Soc Jpn 1985;58:2107-12.

23. Adak A, Pal A, Bandhyopadhyay M. Spectrophotometric determination of anionic surfactants in wastewater using acridine orange. Ind J Chem Technol 2005; 12:145-8.

24. Fendler JH, Hinze WL. Reactivity control in micelles and surfactant vesicles. Kinetics and mechanism of basecatalyzed hydrolysis of 5,5'-dithiobis(2-nitrobenzoic acid) in water, hexadecyltrimethylammonium bromide micelles, and dioctadecyldimethylammonium chloride surfactant vesicles. J Am Chem Soc 1981;103):5439-47.

25. Solntsev KM, Huppert D, Agmon N. Solvatochromism of $\beta$-naphthaol. J Phys Chem 1998;102:9599-606. 